\title{
Synthesis and Chiroptical Study of D-/L-Penicillamine-Capped Silver Nanoclusters
}

\author{
Naoki Nishida, ${ }^{a}$ Hiroshi Yao, ${ }^{a}{ }^{*}$ Tomoyasu Ueda, ${ }^{\mathrm{b}}$ Akito Sasaki, ${ }^{\mathrm{b}}$ and Keisaku Kimura ${ }^{\mathrm{a}}$
}

a Graduate School of Material Science, University of Hyogo, 3-2-1 Koto, Kamigori-cho, Ako-gun, Hyogo 678-1297, Japan

b X-ray Research Laboratory, Rigaku Corporation, 3-9-12 Matsubara-cho, Akishima, Tokyo 169-8666, Japan

\section{Stability of Fractioned Silver Nanoclusters}

Figure S1 shows the UV-visible absorption spectra of compound $\mathbf{2}_{\mathrm{L}}$ as a function of time. The spectra were recorded immediately after extraction (blue curve) and after 24 hours of the extraction (black curve). In compound $\mathbf{2}_{\mathrm{L}}$, although we can observe a well-defined peak at $\sim 480 \mathrm{~nm}$, it decreases with the passage of time, implying that the compound $\mathbf{2}$ is relatively unstable in solution.

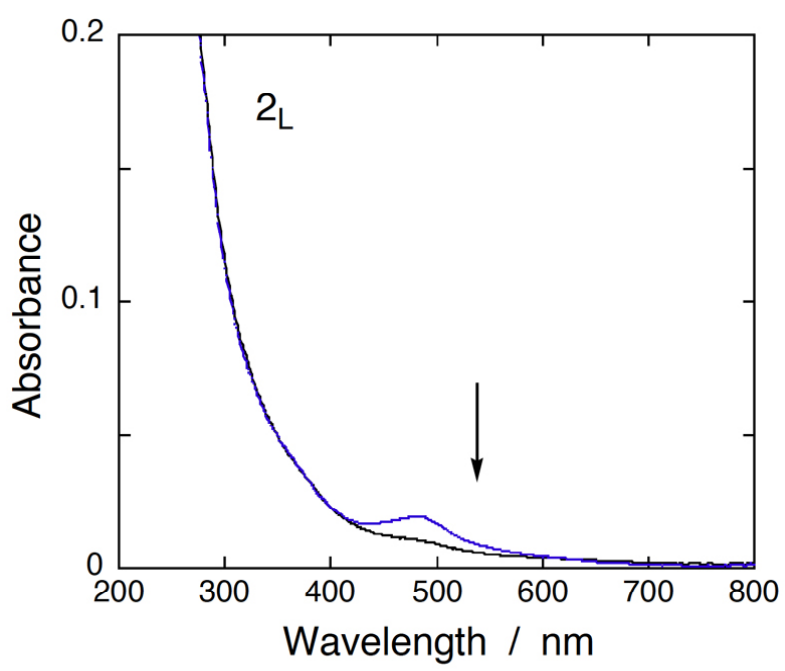

Figure S1. UV-visible absorption spectra of compound $\mathbf{2}_{\mathrm{L}}$ as a function of time. Blue and black traces were recorded immediately after extraction and after 24 hours of the extraction, respectively.

\section{Ordinary Absorption and CD Spectra of L-/D-Penicillamine in Aqueous Solution}

Figure S2 shows the ordinary absorption (left) and CD spectra (right) of L- and D-penicillamine in aqueous solution. These thiols contribute to the CD signals only in the UV region and show a clear mirror image relationship. 

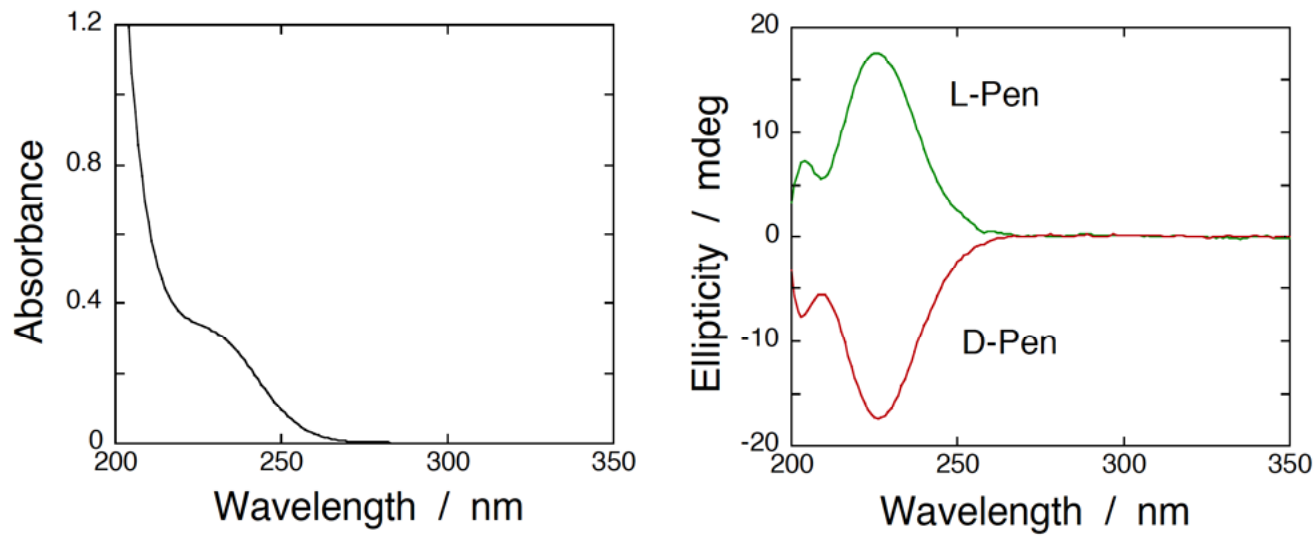

Figure S2. Absorption (left) and CD (right) spectra of pure L-Pen and D-Pen in aqueous solution. No CD signals were obtained for $r a c$-Pen.

\section{Spectroscopic Characterizations of Compounds 4 and 6}

Figures S3-a and S3-b show the absorption and CD (anisotropy factor) spectra of the fractioned compounds $\mathbf{4}$ and $\mathbf{6}$, respectively. Figure S4 shows the size distributions of compounds 4-6 obtained by SAXS. Both compounds (4 and 6) exhibit featureless absorption spectra. The chiroptical response observed in $\mathbf{4}_{\mathrm{L}} / \mathbf{4}_{\mathrm{D}}$ showed a mirror-image relationship. The

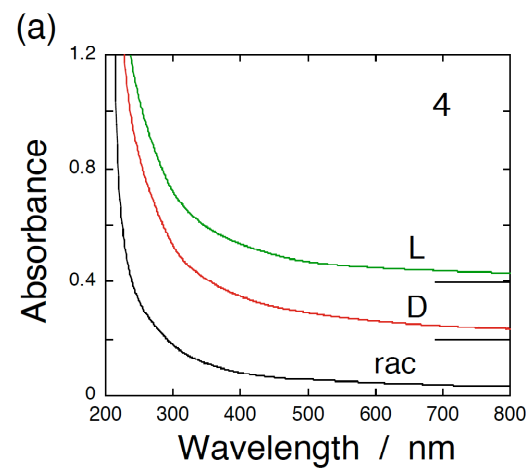

(b)
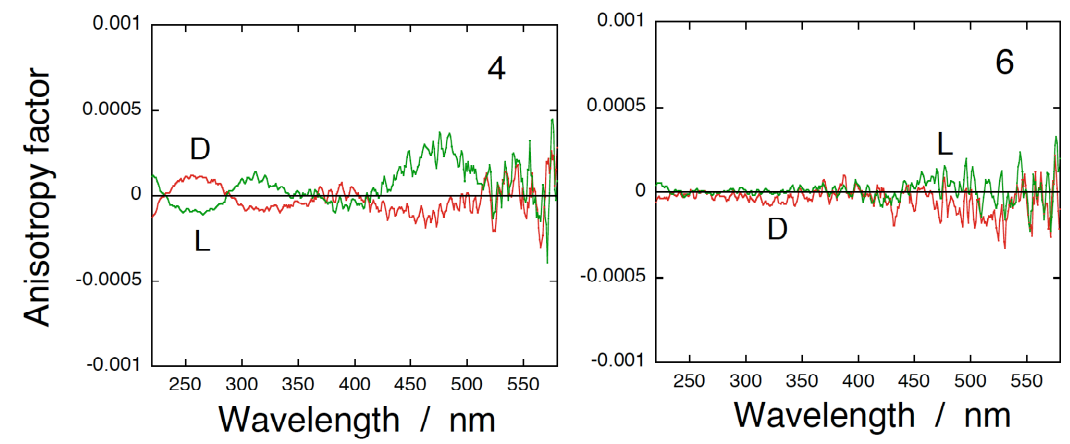

Figure S3. (a) Absorption spectra and (b) anisotropy factors of the fractioned cluster compounds $\mathbf{4}$ and $\mathbf{6}$. compound $\mathbf{6}$ exhibited very weak CD signals. Consequently, the fractions $\mathbf{4}$ and $\mathbf{6}$ follow the spectroscopic trends of the other components.

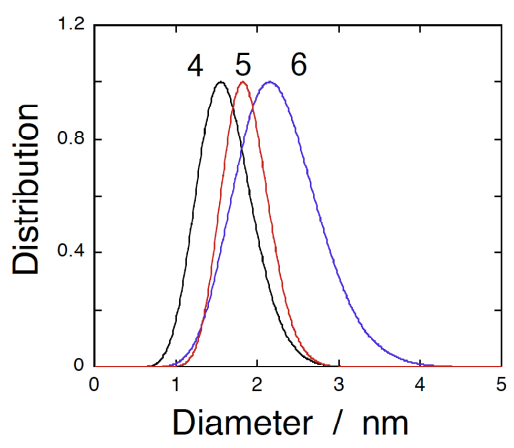

Figure S4. Size distributions of compounds 4-6 obtained by SAXS. 